
\title{
25 Research Square \\ The intestinal microbiota shapes gut physiology and regulates enteric neurons and glia
}

Fernando A. Vicentini

Catherine M. Keenan

Laurie Wallace

Crystal Woods

Jean-Baptiste Cavin

Amanda Flockton

Wendy Macklin

Jaime Belkind-Gerson

Simon Hirota

Keith A. Sharkey

\section{Video Byte}

Keywords: enteric nervous system, ENS, gastrointestinal motility, short-chain fatty acids, SCFAs, lipopolysaccharide, LPS, enteric glia, enteric glial cell, myenteric plexus, submucosal plexus, enteric neuron, gut microbiota, Microbiome, gut function, gastrointestinal tract, GI tract, enteric neuropathy, antibiotic, gut microbes

Posted Date: March 1st, 2022

DOI: https://doi.org/10.21203/rs.3.rs-1405866/v1

License: (c) (i) This work is licensed under a Creative Commons Attribution 4.0 International License.

Read Full License 


\section{Abstract}

The gut microbiome helps regulate gastrointestinal physiology, partly by interacting with the extensive enteric nervous system (ENS). Under disease conditions, gut microbiome alterations are often accompanied by ENS disruption, but it's unclear exactly how the microbiota regulates gut function and the ENS. To learn more, a new study examined the effects of microbiome depletion and restoration in mice. Antibiotic-induced microbiome depletion caused structural and functional changes in the gut. These changes were accompanied by loss of neurons from both ENS layers (the submucosal and myenteric plexuses) in the ileum and proximal colon and by loss of enteric glia from the myenteric plexus in the ileum. Natural microbiome recovery restored gut function and promoted the growth of new neurons and glia. Supplementation with the microbial factor lipopolysaccharide increased enteric neuron survival during antibiotic treatment, while microbial short-chain fatty acid treatment helped restore neuron numbers after antibiotic treatment. Although the mechanisms remain unclear, the results show that the gut microbiome affects gut structure and function as well as the ENS and that specific microbial products can support ENS health, providing new insights that may aid in the development of treatments for ENS pathologies. 